\title{
Child Bearing Decisions among Women Enrolled In PMTCT Programme: A Comparative Study of the Rural and Semi-Urban Setting of Malawi Chiradzulu District
}

\author{
Article by Olive Jean Makuwira \\ Public Health, Texila American University, Malawi \\ E-mail: makuwiraolive@yahoo.com
}

\begin{abstract}
Background: HIV and AIDS have caused untold harm and human suffering globally. Over $90 \%$ of people infected with this deadly virus live in sub-Saharan Africa including Malawi. In Malawi HIV disproportionally affects women in comparison with men. According to Demographic and Health Survey (2015-2016) key indicators, HIV prevalence among women aged 15-64 was $12.8 \%$ compared to men $8.2 \%$ public health. As per 2010 Malawi demographic and health survey, HIV prevalence in the southern region was twice as high as prevalence in the other regions (Southern 14.5\%, Central 7.6\% and northern region (6. 6\%).Women and children are more affected than the rest of the general population. HIV infection in children below 15 years is largely due to mother-to-child transmission (MTCT). Worldwide, over two million children have been infected through MTCT.

Type of Research Study: The study adopted a qualitative research design using the inductive research technique or grounded theory research in which the steps occur simultaneously, observing, collecting data and forming theory from the data at the same time.

Problem Statement: HIV prevalence in Southern Region of Malawi is twice as high as Northern and Central regions, at $14.5 \%$ where fertility rate at $5.7 \%$ are also high. A combination of high fertility rate and high HIV prevalence have contributed to the undermining of an effective PMTCT continuum of prevention, care and treatment programme towards achieving virtual elimination of mother to child HIV transmission and keeping the mothers alive. Therefore, there is need to examine how and through what pathways learning about one's HIV positive status could alter one's childbearing decisions.

Objectives: This study explored the decision-making process on pregnancy decisions among PMTCT participants and their family support systems; Examined factors that influence decision making process among PMTCT participants and their immediate family networks; Determined the gaps in the PMTCT service delivery; Explored the knowledge and attitudes of health providers PMTCT mothers; and analysed the gap between the PMTCT policy guidelines and their implementation

This study revealed factors that influence fertility decisions among pregnant women with HIV attending ANC and ART clinic at Chiradzulu District Hospital. Some of participants became pregnant because they desired to have another child and others because their partners wanted a child. A proper understanding of factors associated with pregnancies among these women is essential to guide interventions and counselling strategies to better inform and support them. Religious doctrines, beliefs about having a healthy baby, personal desire and familial desires to have a baby, combined with the social stigma that accompanies HIV, puts women who are HIV-positive in a difficult situation. HIV-positive women must be appropriately counselled about the risks of childbearing rather than focusing only on issues related to mother-to-child transmission of HIV.
\end{abstract}

\section{Introduction}

The increased availability and access to free antiretroviral medication (ART) for HIV positive pregnant and lactating women has led to fewer children acquiring HIV infection. According to the 2016 Global Plan progress report, there has been a 60\% decline between 2009 and 2015 in the number of children acquiring new HIV infection. Much of this success is attributed to improved antiretroviral regimens to keep children 
DOI: $10.21522 /$ TIJPH.2013.05.04.Art040

ISSN: $2520-3134$

safe from HIV and maintain maternal health; and to the introduction of new ways of delivering services including program integration and expansion.

(Maier, 2008) Concludes that antiretroviral therapy is associated with increased fertility desire, but not pregnancy or live birth, among HIV+ women in an early HIV treatment program in rural Uganda.

Yet despite this progress, the impact of HIV on maternal and child health continues to pose significant challenges in Sub-Saharan Africa. In Malawi for instance, HIV prevalence among antenatal women is currently estimated to be $10.6 \%$, with about 57,000 women living with the disease. Mother to child HIV transmission (MTCT) is of huge concern as it is by far the largest source of HIV infection in children below the age of 15 years. Children acquire HIV from their

Mothers during pregnancy; labour and delivery, or through breastfeeding

Efforts to prevent mother-to-child HIV transmission (PMTCT) have generally focused on treatment scale-up. However, much more yield could be realised with the addition of strategies focusing on contraception. Evidence shows that a strategy focusing on contraception other than ART could avert 28.6\% more HIV positive births. This finding is in line with the WHO public health approach to PMTCT which among its recommended four prongs advocate for "prevention of unintended pregnancies among HIVpositive women". There are within this package element of improving access to Family Planning (FP), providing voluntary counselling and testing to clients accessing FP, and supporting HIV-positive women in making informed decisions about their reproductive lives.

The fertility rate in Malawi is estimated to be 4.4; with only $43 \%$ of women of child bearing ages (1549 years) reported using modern contraception. About $23 \%$ of the pregnancies among HIV positive mothers are observed to be unintended. The need to combine PMTCT and FP is therefore imperative.

Previous studies have documented a decreased desire among HIV positive women to have more children compared to their HIV negative counterparts. Yet this desire appears to reduce with overall improved health status of HIV-positive women. Interestingly still, the latter fact stands in contrast to the finding by (Maier, 2008) who concludes that antiretroviral therapy is associated with increased fertility desire, but not pregnancy or live birth, among HIV+ women in an early HIV treatment program in rural Uganda.

Studies have further documented the important role played by socio-cultural factors in influencing fertility decisions, which among others include HIV status and ART use. The present study seeks to obtain in-depth understanding of the fertility decision among HIV positive women attending their PMTCT services in Chiradzulu district. This is one of the areas where there has been a large roll out of the ART Programme and where PMTCT interventions were first started.

\section{Background information}

HIV and AIDS have caused untold harm and human suffering globally. Over $90 \%$ of people infected with this deadly virus live in sub-Saharan Africa including Malawi. In Malawi, the prevalence of HIV among the childbearing age group (15-49) is estimated to be at $14 \%$ and close to a million people have already died of AIDS-related conditions. Women and children are more affected than the rest of the general population. HIV infection in children below 15 years is largely due to mother-to-child transmission (MTCT). Worldwide, over two million children have been infected through MTCT.

The high prevalence of HIV among the women is contributed from multiple factors. The factors that are responsible for the proliferation of HIV and AIDS in these women include tradition, gender inequalities, biological make up, inadequate coverage of health care in some areas, distance, failure of women to negotiate on matters regarding sex and poverty among others. It is estimated that $18 \%$ of the pregnant women in Malawi are infected with HIV and this prevalence is relatively high in urban areas as it ranges from 19 to $30 \%$. As a result of this, MTCT of HIV becomes an area of concern and calls for the prevention of MTCT (PMTCT) of HIV.

In Malawi, PMTCT was first introduced in 2001 into the ANC as an international initiative and then was adopted by different countries in Africa. The primary aim of the PMTCT programme, as put in the guidelines, was to decrease the number of HIV-infected babies born to HIV-positive mothers. Primary 
prevention of HIV infection, particularly among women of childbearing age, has always been the backbone of the programme. It is believed that without intervention, up to $50 \%$ of babies born from HIV-positive women get infected through labour, delivery and breast feeding. A number of interventions have been aimed at limiting the risk of new-born infections during delivery, such as caesarean section as the mode of delivery and administering antiretroviral (ARV) drugs prepartum and peripartum and providing alternatives to breast feeding. However, all these approaches are not always possible in developing countries and the relative importance of ARV drugs, in particular Neverapine (NVP), has been investigated.

HIV testing starting with 'opt-in' strategy was introduced in PMTCT programs in 2001 following international initiatives, with efforts to reduce MTCT. Opt-in strategy is an approach whereby all clients are encouraged to undergo voluntary counselling and testing (VCT) at their own will. Later, opt-out strategy was adopted by many countries in sub-Saharan Africa. The aim was to enable all pregnant women to be screened for HIV infection. Screening should occur after a woman is notified that HIV screening is recommended for all pregnant women and that she will receive an HIV test as part of the routine panel of prenatal tests unless she declines (opt-out screening).

PMTCT programmes adopted different policies to ensure successful intervention of the programmes. In 2002, the MTCT-plus Initiative was established, which aimed to move beyond interventions to prevent infant HIV infection. It does this by supporting the provision of specialised care to HIV-infected women, their partners and their children who are identified in MTCT programmes.

Much attention has been given to the ARV prophylaxis in developing countries. Commonly a single dose of NVP $200 \mathrm{mg}$ administered to a woman at the onset of labour and a similar drug, but in syrup form given to the child within 72 hours after delivery reduces infection by $38-50 \%$. The single use of NVP as prophylaxis in PMTCT has been recommended by the World Health Organisation (WHO) and widely adopted by many resource-poor countries particularly those in sub-Saharan Africa. The availability of NVP and its simplicity in administration including the fact that it is cheap with fewer side effects make it acceptable, accessible and affordable in resource-poor settings. However, the single use of NVP as prophylaxis in PMTCT programmes has been associated with the development of resistance at some stages in the lives of women who eventually receive ARV treatment. According to Kasenga (2010), it is for this reason that a combined regimen has been adopted; although at the time of conducting the study, only NVP was being used in PMTCT.

\section{Literature review}

\section{Introduction}

This section summarizes existing literature on reproductive health decisions by HIV positive women. Studies done on the social cultural and policy issues regarding PMTCT are further discussed. These are presented under the following sub-headings;

\section{Knowledge perceptions, attitudes of PMTCT service providers}

Studies have shown that health worker attitudes to some degree mediate HIV positive women's decisions about their reproductive health choices. In their study on providers attitudes and knowledge of safer conception for instance, (Kawale, 2015) asserted that providers (health workers) have complex attitudes about HIV-infected clients having children. They further assert that health workers lack specific knowledge to appropriately counsel clients about reproductive health and safer conception strategies; have low level of knowledge of reproductive health, the efficacy of PMTCT, and the risks of pregnancy for HIV-infected women. The authors go on to assert that health workers are ambivalent about supporting childbearing among their clients with HIV and have raised concerns about HIV-infected individuals continued child bearing.

Similarly, (Gombachika B. E., 2013) assert that despite the global reports about health worker's perceptions, and preferences and values consciously or unconsciously influencing the choices available to $\mathrm{PLWH}$, there is little by way of systematic understanding of reasons for the problem. The authors further 
DOI: $10.21522 /$ TIJPH.2013.05.04.Art040

ISSN: $2520-3134$

documented PLHWs describing health workers to be "judgmental and reacting negatively to their marriages and child bearing".

(Nyasulu, 2011) Asserted that there is lack of involvement in decision making process regarding access to reproductive health services such as PMTCT services for HIV positive pregnant women.

2 Policies and Guidelines on Prevention of Mother to Child Transmission (PMTCT)

Malawi's integrated antiretroviral treatment (ART) / PMTCT programme commenced in 2011 following the adoption and adaptation of the 2010 World Health Organization (WHO) recommendations. WHO public health approach to PMTCT includes four prongs which are: preventing new HIV infections among women of childbearing age; preventing unintended pregnancies among women living with HIV; preventing HIV transmission from a woman living with HIV to her baby; Providing appropriate treatment; care and support to mothers living with HIV and their children and families (WHO, 2011).

A study done by Gombachika et. al., 2013 showed that health workers attitude, weak linkages between HIV/AIDS and sexual reproductive health services, contradictory messages between media and hospitals and lack of information are directly related to guidelines and policies. The study emphasizes the need for updating policies and guidelines in order to provide services to couples living with HIV in Malawi (Kasenga, 2010).

(Njunga J., 2010) Showed that in the context of customary matrilineal kinship, matrilocal residence patterns and complete male absence from the PMTCT program, the demand by the PMTCT program for partner disclosure played up fears of rejection among men. Further, given accusations of infidelity by the wives' relatives, this situation that led many men to abandon their families.

This scenario strengthens another view by (Gombachika B. E., 2013) that the policy guidelines implemented with obvious public health interests in mind sometimes directly conflicts with the living reality of the PLWHA. The authors concluded that, "Surprisingly, the HIV, AIDS and sexual and reproductive health policy agenda in Malawi is not considering these issues and does not reflect the social cultural context for couples living with HIV".

\section{Community support structures and networks}

Globally, studies on pregnancy decision making among HIV -positive women acknowledge the huge impact of social processes and systems within which decisions are made. The studies observed that social dynamics encourage child bearing or stifle the desire for motherhood. Collectively they represent hard reproductive choices that are made under profound social constraints (Chi Kim Bui, 2011) leading to another concept, that the "concept of reproductive choice itself" needs interrogation.

Sheri B. Kirshenbaum et al (2004), highlighted a desire for motherhood; stigma; religious values; attitudes of partners and health care providers; and the impact of the mother's health and longevity on the child (Iwelunmor) as some of the key psycho- social factors.

The International Community of Women Living with HIV/AIDS report (2015), again cited negative social reactions for continued child bearing among HIV positive women. In their study (Kaida, et al., 2011) reveal that same socio-economic factors as being drivers to continued child bearing. For instance, South Africa a study on the 'Child bearing Intentions of HIV-positive women of reproductive age in Soweto, highlighted the fact that there is stigma associated with childlessness in many societies even though potential health risks may have dampened the fertility intentions of some HIV-positive women.

An ethnographic survey exploring the gendered influences on women's uptake of ANC services in Punjab, Pakistan, shows that $33 \%$ of Punjab women report that the use of ANC is associated with strong influence of mothers-in-law in the decision making process regarding pregnancy.

Biseck, et al., (2015) in their assessment of the cultural and social factors associated with fertility decisions in WLHA, concluded that a proper understanding of factors associated with pregnancies among these women is essential to guide interventions and counselling strategies to better inform and support them. Religious doctrines, beliefs about having a healthy baby, personal desire and familial desires to have a baby, 
combined with the social stigma that accompanies HIV, puts women who are HIV-positive in a difficult situation.

The emerging picture from studies point to the fact that the questions about the drivers behind pregnancy decisions are a complex web of socio-cultural and contextual that cannot be fully understood by a single approach.

Social cultural effects on reproductive health decision making.

King et al, (1995), found out some of the reasons for HIV pregnant woman to become pregnant were emotional and personal reasons. The women cited their partner's desire as an important factor of becoming pregnant which was often intercultural expectation of child bearing. The results also shows the desire for a male child or heir. New partner and religion also contributes to the decision making (Kannaippan, 2012).

Rutenberg, et al., (2000) conducted a study on Reproductive decision making in the context of HIV and AIDS which shows that in the absence of signs and symptoms of HIV, reproductive health decision making is generally weak including family planning. However, when signs and symptoms of the disease are present, both men and women are overwhelmingly against continued childbearing.

Kannaippan, et al. (2012), in a study finding shows that the major factors distinguishing women who wanted to have children and those that did not was anxiety. Women, who did not have family support, were reluctant to opt for pregnancy as they were not sure of the future. Similarly, Iwelunmor (2008), shows that there is an implication of family support in child bearing decisions.

It is clear that there is a gap as to the understanding of the impact of social networks, families and husbands on child bearing decision of HIV positive women in the implementation of the PMTCT services.

\section{Summary of literature review}

From the review of the literature the observations outlined below justifies carrying out the study. They contain both gaps inherent in the previous studies as well recommendations for further investigation to improve our understanding of the context in which the child bearing decisions by WLHA are made. Collectively, these factors present the broad structure or framework from which the study will be designed and the research questions addressed.

Despite the comprehensive PMTCT guidelines, there is still more that can be done in the context of Malawian policies to help HIV positive women in making child bearing decision. These could be in form of improving in the clarity of the information to reduce the incidences of contradictory messages between media and the hospitals, strengthening the monitoring systems and increasing the availability of information resources.

Review of literature suggests that PMTCT providers would do well to scale up elements of prevention of unintended pregnancies pillar in the WHO. Strong evidence points that more effort is devoted to treatment aspect at the expense of preventive strategies advocated for in the Malawi Integrated Guidelines for Providing HIV Services (Ministry of Health, 2011)

The WHO recommendations while comprehensive enough need to be thorough reviewed for consistency with our local context. The issues of capacity of our PMTCT service providers including information resources and knowledge levels need to take into account. This will be very important in assisting the providers change their mind-set. This comes as it appears that striking a balance between public health interests and respecting the sexual \& reproductive rights of PLHWA is a challenge.

Synchrony between ART Services, PMTCT, Sexual and reproductive health rights and Family planning in general is weak at operative level. An examination of the constraints and opportunities of integrating these services at operation level can provide a good entry point. This would help to develop ways of strengthening the weak linkage between HIV, AIDS and sexual and reproductive health services.

The policy implementation so far appears to overlook the forces in the social cultural environment in which the child bearing decisions. The service providers appear not to understand or choose to ignore the socio economic pressures that the PLWHA contend with in making these decisions. 
DOI: $10.21522 /$ TIJPH.2013.05.04.Art040

ISSN: $2520-3134$

There is need to look at the community and family support networks available to support the PLWHA. The literature is deficit on strategies that PMTCT, ART and Family Planning programmes employ to engage other stakeholders at the community.

The literature appears to have the scanty information available on comprehensive studies done to understand the knowledge, attitudes and skills of providers of PMTCT. This would assist in improving our understanding of the continuum in which learning and pathways about child bearing among PLWHA takes place. One key skill-set to focus on should be psychosocial support and counselling.

Taken together these shortcomings make a case to conduct this study exploring these three targeting these three areas: Policy shortcomings, constraining socio economic drivers and ineffective barriers a the service provision level.

\section{Methodology}

\section{Research design and methodology}

The study adopted qualitative research design using the inductive research technique or grounded theory research... An important aspect of this methodology is the constant comparative process between pieces of data (Burns, 2009).

\section{Study setting}

The study was conducted at the antenatal clinic in Chiradzulu District in the Southern Region of Malawi. The district has a population of 246,489 out of which 153,752 are females. The district shares boundaries with Zomba, Blantyre, Phalombe and Mulanje.

The site was chosen because of the high prevalence rate of HIV in the district which is currently at $17 \%$ (Ministry of Health, 2014) and long history of implementing PMTCT programmes as the health facility is among the pioneer implementers of programmes in Malawi.

\section{Study population}

In this study, the target population was all HIV positive women who are in the PMTCT program at Chiradzulu District Hospital, their immediate and extended family network.

\section{Research objectives}

\section{General objective}

To explore the decision making process on pregnancy decisions among PMTCT participants and their family support systems

\section{Specific objectives}

A. To examine factors that influence decision making process among PMTCT participants and their immediate family networks

B. To explore the knowledge and attitudes of health providers PMTCT mothers

C. To analyse the gap between the PMTCT policy guidelines and their implementation

\section{Research questions}

The study was guided by the following questions:

1. What factors influence pregnancy decision among PMTCT participants

2. What support is offered to PMTCT women and their couples when making pregnancy decisions

3. To what extent does social cultural environment influence pregnancy decisions among PMTCT participants and their spouses

4. Are there gaps in the PMTCT implementation, guiding policies; or service provision capacities and attitudes by health providers? If so, what is being done to fill the gaps? 


\section{Data collection analytical framework \& reporting}

The study utilized a combination of methods: records, in depth participant interviews, key informant interviews and focus group discussions.

3.6.1 Validity: Triangulation

The study used data triangulation technique. Triangulation is the combination of methodologies in the study of the same phenomena or programme. (Patton, 1990).

3.6.2 Data Collection Instruments

Three set of instruments were used as follows:

3.6.2.1 Focus group interviews with participants and community.

A semi structured, coded questionnaire with close ended and few open ended questions was used to collect data from participants. The development of the data collection instrument was influenced by possible factors that may contribute to HIV positive women's decisions on child bearing

3.6.2.2 Interview guide for key informant interviews

Semi structured interview guide for use with key Informants including health workers and PMTCT programme coordinators

3.6.3 Pretesting the Data Collecting Instrument

Pretesting helped to determine whether the proposed study was feasible, and to examine the reliability and validity of the research instrument (Burns, 2009). The instrument were refined following the pretesting as they were also used in pretesting which was done in South Lunzu Health Centre in Blantyre. This was because of the similarities in setting and service provision on PMTCT offered at South Lunzu Health Centre and Chiradzulu District Health office, pretesting at this facility provided a good guide.

3.6.4 Sampling Framework

Purposive sampling was used to select interviewees who meet the inclusion criteria at the antenatal clinic. Purposive sampling is a non-probability sampling method in which the researcher selects participants based on personal judgment about who will be most representative or informative (Polit, 2010). Participants were informed about the study and were required to sign a written consent before the interview.

\section{Inclusion criteria}

Participants were categorized as follows:

A. 15 women who were diagnosed while on antenatal care

B. 15 women received diagnosis prior to getting pregnant

C. PMTCT participants with children

D. 15 PMTCT participants who have not yet given birth

E. Four focus group discussions were arranged in two villages around the district. The arrangements were coordinated by the PMTCT at the District Hospitals.

Participants (volunteers) were selected $\mathrm{b}$ based on their knowledge and involvement with health or PLWHA issues (support groups, CBO etc) in the community, spouses or extended family members of PMTCT participants.

\section{Data collection}

Data was collected from face to face interviewing the respondents in a private room. Appropriate places were agreed upon with Hospital officials on venues for focus group discussions and in the communities themselves.

The researcher used interview with the aid of a question guide. The question guide included both close and open ended questions (semi structured and structured questions). It was be formulated in English, but it was translated in Chichewa for easy communication with the participants. The interview method was chosen because it had a distinct advantage in that, it enabled the researcher to establish a rapport with participants and gain their cooperation enabling collection of rich information. In addition, interviews, allowed the researcher to clarify ambiguous answers and when appropriate, seek follow up information. 
DOI: $10.21522 /$ TIJPH.2013.05.04.Art040

ISSN: $2520-3134$

Structured questions easily accommodate large number of people. Furthermore, people are more truthful while responding to question guides regarding controversial issues in particular due to the fact that their responses are anonymous.

\section{Computer software and data analysis}

According to (Creswell, 2011), a researcher needs basic skills in analysing data including coding text and developing themes and descriptions based on these codes and should be acquainted with qualitative data analysis software package. In this study the researcher used thematic analysis which involves clustering together related types of narrative information into a coherent scheme. Through inductive reasoning process, the researcher starts by coding the data, assigning a label to each unit and then building the codes into themes. The researcher used SPSS software program to analyse the data

\section{Dissemination of findings}

The study results have been disseminated to Chiradzulu District Hospital, Ministry of Health and Texila University.

\section{Limitations of the study}

Being qualitative in nature, the study did not have the advantage of highly rigorous quantitative design that allowed extrapolation of the findings or replication. However, the qualitative approach was adopted due to the strongly sensitive personal and emotive nature of the subject hence need for in depth interviewing.

\section{Ethical consideration}

Approval to conduct the study was sought from College of Medicine Research Ethics Committee (COMREC). Permission was obtained from the District Health Officer of Chiradzulu to conduct a study at the hospital.

\section{Data analaysis}

\subsubsection{Introduction}

The analysis of qualitative data was done using Nvivo but SPSS was used for socio-demographic data and knowledge and attitudes about PMTCT frequencies

\section{Data organization}

Characteristics of participants assessed include age. The second part covers the qualitative data as follows: To examine factors that influence decision making process among PMTCT participants and their immediate family networks. To explore the knowledge and attitudes of health providers PMTCT mothers. To analyse the gap between the PMTCT policy guidelines and their implementation. No name was used, transcripts were labelled as participant $\mathrm{A}, \mathrm{B}, \mathrm{C}$ to $\mathrm{Z}$ corresponding to participant $1,2,3 \ldots 26$. Comments and reports from participants were presented according to the responding participant. 


\section{Demographic characteristics of participants}

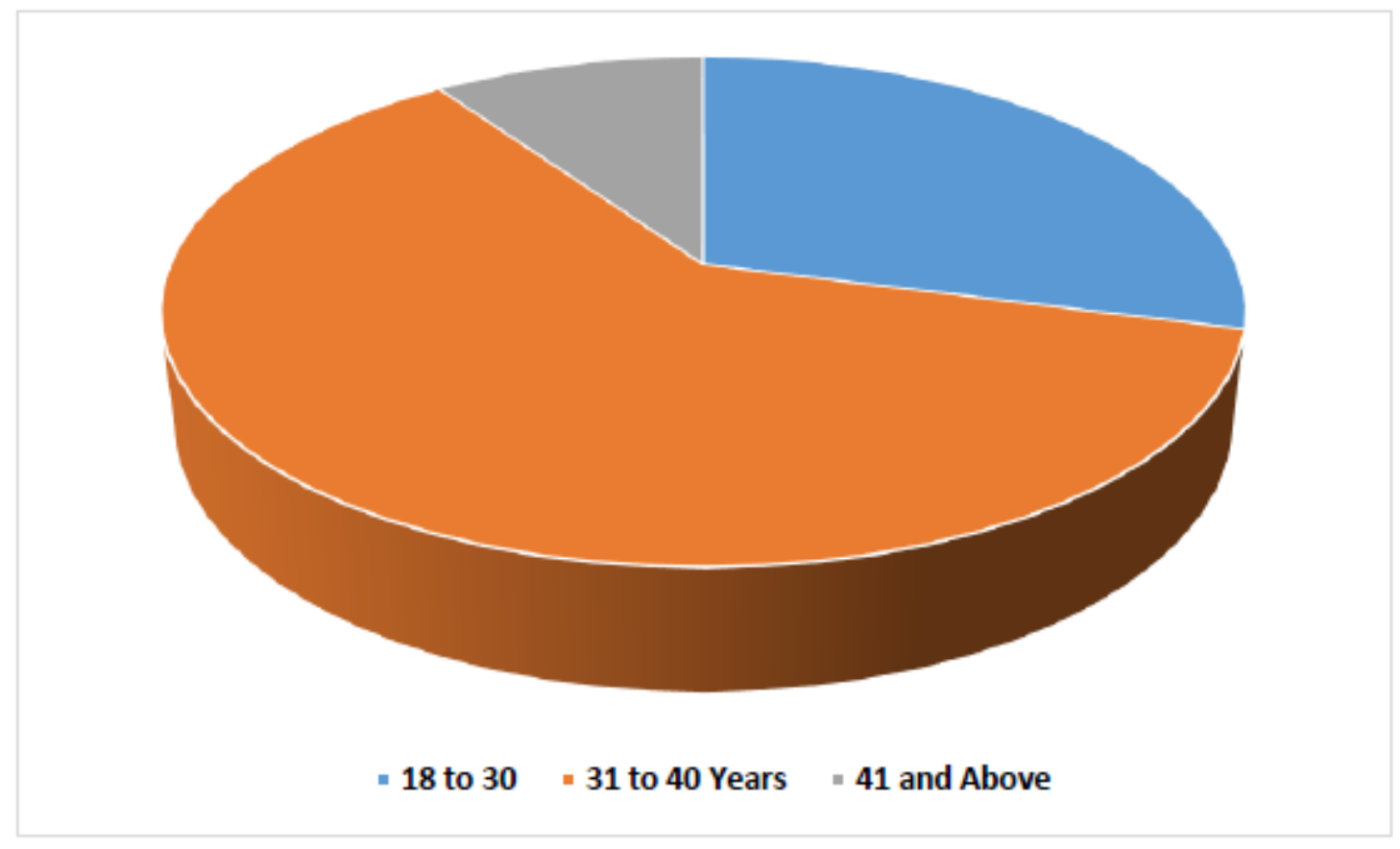

Figure 1. Frequency distribution of participants by age (years)

From the total number of $(n=53)$ participants, the minimum age was 18 years and maximum age was 42 years. The average age of the participants was 32 years and the majority of participants are aged between 31 to 40 years old, followed by those who are 18 to 30 years old.

\section{Influences on childbearing}

Majority of respondents had good knowledge and good understanding of the PMTCT program. They understood how the PMTCT program works and its importance in preventing mother-to-child HIV transmission. About 17 respondents reported that their decisions to join PMTCT program was based on the preventing of their unborn babies from being infected.

Some of the respondents said they do not know much but they have some knowledge about the PMTCT program. They joined PMTCT program because of counselling from health professionals or relatives or friends. Some respondents joined PMTCT program because they wanted to gain more knowledge about PMTCT program

We tried to establish the influences on childbearing among WLHA at Chiradzulu District Hospital. All 53 participants agreed that there were multiple influences. Some of the influences pertained to their spouses. Two participants, who were aged 27 and 30 years, respectively, said: "I wanted to please my husband." Because their husband really wanted a child at all cost

For some, their husbands were looking for either a male or female child. Some women reported that they feared marital breakdown if they did not bear children. Some of the respondents said that they planned to have children because their status does not limit them from bearing children, as there are drugs available to protect their children. They wanted to bear children and prove to others that they can also produce since there are messages disseminated in the media regarding childbearing among HIV-positive women.

Four participant who were aged 26 and 35-year-old participant said:

"They were influenced by the Drama group that came in their villages and the messages was HIVpositive people are free to bear children but adhere to drug therapy to prevent transmitting HIV to the new baby." 
DOI: $10.21522 / \mathrm{TIJPH} .2013 .05 .04 . A r t 040$

ISSN: $2520-3134$

Another participant, aged 32 years, opened up to say:

"I was influenced by the health worker at the hospital because I was offered medicine which can help me live happy and healthy but also the medicine protects the child to from HIV."

However, some reminded us that pregnancies are sometimes unplanned. This was evident by what one 21-year-old participant said:

"I just found myself pregnant. I was on contraceptives but on a certain month, I missed my menses. It was very disappointing."

Another response, from a 39-year-old participant, was:

"I do not know what made me to bear children. I just wanted to bear children."

Three participant aged from 21 and 37-year-old, said:

"I needed a child most because a health worker told me that though I am positive and on ART $i$ can I have a child and bear a child without virus unless I get myself enrolled on PMCTC.",

Although the number of children to be borne by every participant differed, the categorised data showed responses such as, offered by a 38-year-old participant:

Fourteen participant between 23 and 40 years said:

"I was advised to stop giving birth after three children"

Another response, from a 27-year-old participant, was

"I will stop giving birth after two children"

Social cultural environment influence pregnancy decisions among PMTCT participants and their spouses

It is therefore particularly difficult for married women or those intending to get married, regardless of their HIV status, to exercise a choice not to have children. Men appeared to be less pressured by family and societal expectations regarding parenthood. This underscores the gender power imbalances in intimate sexual relationships between women and men as well as the relatively less powerful position women occupy in society. There are apparent contradictions in the social pressures HIV-positive women and men experience regarding reproduction. Whether or not individuals who disclosed their HIV positive status, it surfaced as a key factor in the social pressures they encountered.

On the one hand, women who were married and who had not disclosed their HIV status felt pressured to have children to avoid raising community suspicions regarding their HIV status.

This is an important consideration for participants in a setting in which being HIV positive is stigmatized (Centre for Social Science Research, 2002). On the other hand, seeking to have children for women and men who had disclosed their HIV positive status was socially unacceptable. This is supported by evidence from another study that showed considerable stigmatization of childbearing by HIV positive women among the general populace in this setting (Myer et al., 2006).

Women found themselves particularly pressured by such attitudes, and this may have acted as a constraint to disclosure of their HIV positive status.

This survey of HIV positive women revealed a dichotomy between personal and perceived societal views on pregnancy and childbearing in HIV positive women, with more than half of the participants experiencing societal pressure to forego childbearing." These results highlight the need for up-to-date, factual education regarding pregnancy and childbearing in HIV positive women among both the lay and medical communities.

According to the study conducted by Sowell (1997), there were six themes related to reproductive decisions namely: spiritual and religious beliefs, knowledge and beliefs about HIV, previous experience with childbearing, attitudes of families and sex partners, personal health, and intrapersonal motivation to have a baby. In the same study, one group of women indicated that they had fulfilled their desire to bear children prior to diagnosis; while a second group had experienced the illness or death of a child with HIV and was weary of additional pregnancies. Additionally, a third group of women indicated they would consider an accidental pregnancy, but they had no particular desire to become pregnant. Health interventions, such as PMTCT and antiretroviral therapy, could have an important influence on women's 
and men's reproductive decisions, as they affect two important considerations around child's and parent's health, respectively.

\section{Types of support given to pregnant HIV-positive women who are on ART}

Most of the participants had support from the community, and from the hospital they normally visit

In some cases, it was found that the programmes in the community normally gives them Likuni Phala, Mosquito nets and cover other daily needs. Health workers also counsel, especially on the number of children to bear as reported by one participant:

"The health workers advised me not to have many children if I have to live a healthy life."

In other cases, parents advise their daughters not to bear more children while HIV-positive. Participants gave statements like:

"They are saying the number I have is okay because I am HIV-positive."

"They just encourage me not to have any more children, as I am now sick so I have to take care of myself."

Some husbands have been found to be supportive. In such cases, the husbands take the responsibility of providing daily needs:

"My husband buys me whatever I ask him. At some point he escorted me when coming to the hospital; he also bought a mosquito net special for the expected child," said a 32-year-old mother.

Not only do the parents and husbands provide support, but so do the community and society. Some village head men HIV-positive pregnant women and people living with HIV Aids to have good health. Community groups conduct nutrition education through drama for HIV positive people. This was evident from this quotation:

"In our area village headman encourages us that every HIV woman should visit the hospital in time and deliver at the hospital not home."

While most women received support, few number indicated that they do not receive any support. A 28year-old said interviewee said:

"My husband pays very little attention to me. He just knows how to produce children with me... I am not given any chance and sometime am told to stop taking medicine."

"They are side-lined to the extent of separate bed if its couple."

"The Marriage breakdown if woman found Positive."

"Those with Positive people prefer to cook on their own even the use of utensils separately with people."

Some women expressed poor community support being related to stigmatization of people living with HIV:

"Yes, I see some women in the village laughing whenever I pass close to them. Nobody supports me.

"They cannot do any personal development on their own, and they cannot help the community anymore.",

"They are side-lined from the community since they cannot contribute to the development since they are HIV Positive."

Some women: reported that having knowledge of the positive effects of PMTCT on infant health, men were more favourably disposed towards having children, regardless of the availability of ART. Conversely, among women, PMTCT in combination with the availability of ART influenced future reproductive intentions, indicating the important links for women between preventing children's infection and maintaining their own health and ability to care for children.

\section{Knowledge and attitudes about PMTCT}

One of the findings of this study is that majority of respondents (41) were knowledgeable about HIV infection and able to understand what it means to be HIV-positive. Most of the women (24) who knew their HIV status, had disclosed their HIV status (HIV-positive) to their family members and twenty-one participants had disclosed their HIV status to their partners. This contradicts the findings of Myer et al. (2006) who reported considerable stigmatization of childbearing by HIV-positive. Women found 
DOI: $10.21522 / \mathrm{TIJPH} .2013 .05 .04 . A r t 040$

ISSN: $2520-3134$

themselves particularly pressured by such attitudes, and this could have acted as a constraint to disclosure of their HIV positive status. The disclosure has not been a big issue as the results showed in this study, majority of participants supported the idea of disclosing their HIV status either to their family or to their partners. Thus, the majority of the repeat pregnancies for HIV-positive women were both unplanned.

\section{Discussion and recommendations}

Several factors influencing fertility decisions among pregnant women on ART were brought to light by the women in Chiradzulu District Hospital. The majority of the women interviewed became pregnant after they had known that they were HIV-positive. Common factors associated with pregnancy included the husband's influence and the availability of drugs that maintain a good health status and help prevent vertical transmission of HIV. Many women indicated that they felt stronger than before starting ART and that they desired more children. They had hope that becoming pregnant would do them no harm.

It has been revealed in other studies that most HIV-positive women who become pregnant, or desire children after their diagnosis, seem more confident in the efficacy of risk reduction strategies. However, the findings of this study suggest that, regardless of a woman's pregnancy experiences or intentions, reproductive decision-making of participants considers the perceived risks of vertical transmission (which is often overestimated); beliefs about vertical transmission risk-reduction strategies; desire for motherhood; stigma; attitudes of partners and healthcare providers; and the impact of the mother's health. Some women reported that they became pregnant because they wanted or desired to have another child, even though they knew that they were HIV-positive. This is contrary to a study in which many women feared getting pregnant if they suspected that they were-HIV positive, because pregnancy would "bring out" the disease.

In this study, most of the participants reported the desire to have more than three children. These characteristics possibly made the women less empowered to make their own decisions regarding childbearing or contraception, and thus become pregnant unwillingly. In other words, this vulnerability could lead to unstable relationships in which women enter relationships men with intention of receiving support, and this can contribute to repeated pregnancies.

Most of the women had disclosed that they were HIV positive to their husbands only, friends, and family members. Women in a study felt hesitant to disclose their HIV-positivity to others out of fear of stigmatization.

Women were found to feel pressured by such attitudes, and this could have acted as a constraint to disclosure of their HIV-positivity. The present study indicates that disclosure was not problematic, as the results showed that the majority of participants supported the idea of disclosing, either to their families or to their partners. The women discussed being able to participate in social activities more freely and actively. Their openness assisted them in discussing more of their family future through acquisition of knowledge from others. This is similar to a study in which the results showed that repeated pregnancies were more likely to occur among women who did not disclose to their spouses.

Through ANC and ART clinics, where health workers, especially nurses and health surveillance assistants (HSAs) provide health talks. Most women were not consistent with any method mentioned and some even failed to use any method. This agrees with the results of a study on pregnancy desires and contraceptive knowledge and use among HIV positive women for prevention of subsequent pregnancies, where most of the HIV-positive women spontaneously interrupted contraceptive methods, and hence more women had unplanned pregnancies.

It was shown in this study that there is poor support by family, partners, and peers related the reproductive decisions of HIV infected women. This concurs with a study that revealed a lack of support from partners, unequal gender relations, and stigmatization as results of increasing pregnancies amongst HIV-positive mothers, contribute to failure of drug collection in the ART clinics. The same study also found that women who were on ART were the most stigmatized after frequent pregnancies, and this compromised their health.

Another important finding in this study was that the majority of participants stated that their family advised or encouraged them to continue bearing more children. This concurs with other studies on 
reproductive intentions among HIV-positive women, which point out that familial, spousal, and societal expectations for childbearing are important influences on women's reproductive intentions. Families often expect that marriages should give rise to children, and this influences HIV-positive couples to bear children.

\section{Conclusion}

This study revealed factors that influence fertility decisions among pregnant women with HIV attending ANC and ART clinic at Chiradzulu District Hospital. Some of participants became pregnant because they desired to have another child and others because their partners wanted a child. A proper understanding of factors associated with pregnancies among these women is essential to guide interventions and counselling strategies to better inform and support them. Religious doctrines, beliefs about having a healthy baby, personal desire and familial desires to have a baby, combined with the social stigma that accompanies HIV, puts women who are HIV-positive in a difficult situation. HIV-positive women must be appropriately counselled about the risks of childbearing rather than focusing only on issues related to mother-to-child transmission of HIV.

\section{Limitations}

Despite the important roles carried out by husbands and partners in this context, males were not interviewed. There is a need to triangulate results from interviews with women with interviews with men to verify or dispute the points made by each group. The authors recommend further research involving partners of HIV-positive women who attend ANC services. Furthermore, the purposeful sampling method of recruiting participants means that data were collected from unrepresentative study informants, and the results cannot be generalized to a wider population. However, the qualitative data collected was relevant to the study topic.

\section{References}

[1]. Biseck, T. Et. al. (2015). Exploring Fertility Decisions among Pregnant HIV-Positive Women on Antiretroviral Therapy at a Health Centre in Balaka, Malawi: A Descriptive Qualitative Study1. Balaka: Not Published.

[2]. Burns, N. Et. al. (2009). The Practice of Nursing Research: Appraisal, Synthesis and Generation of Evidence (6th Ed). St Louis: Saunders Elsevier.

[3]. Chi Kim Bui, Et. al. (2011). Pregnancy Decision-Making among HIV Positive Women in Northern Vietnam: Reconsidering Reproductive Choice. Antropology and Medicine.

[4]. Cooper, D. Et. al. (2009, June 13). Fertility Intentions and Reproductive Health Care Needs of People Living with HIV in Cape Town, South Africa: Implications for Intergrating Reproductive Health and HIV Care Services. pp. 38-46.

[5]. Creswell, J. Et. al (2011). Designing and Conduction Mixed Methods Research. SAGE Publications.

[6]. Crookes, P. Et. al (2004). Research into Practice: Essential Skills for Reading and Applying Research in Nursing and Health Care (2nd Ed). Edinburgh: Bbailliere Tindall.

[7]. (Ed), D. Y. (n.d.). Skills for Reading and Research in Nursing and Health are. In Handbook of Qualitative Research (2nd Ed) (pp. 105-117). Califonia: Thousand Oaks.

[8]. Gerrish, K. Et. al (2010). The Research Process in Nursing. (6th Ed). West 8: Sussex Wiley-Backwell.

[9]. Gerrish, K. Et. al (2010). The Research Process in Nursing.(6th Ed). West 8: Sussex Wiley-Backwell.

[10]. Gombachika, B. Et. al (2013). Reproductive Decision of Couples Living with HIV in Malawi: What Can We Lean for Future Policy and Research Study? Malawi Medical Journal, 65-71.

[11]. Gombachika, B. Et. al. (2013). Source of Information on HIV and Sexual and Reproductive Health for Couples Living with HIV in Rural Southern Malawi. AIDS Research and Treatment, 11.

[12]. Guba, E. G. (1994). Competing Paradigms in Qualitative Research. New Delhi: Sage Publications.

[13]. Guba, E. G. (2010). The Paradigm Dialog. SAGE Publications.

[14]. International Community of Women Lliviny with HIV/AIDS (2015). Positive Women: Voices and Choices Zimbabwe Report. Harare: ICW. 
DOI: $10.21522 /$ TIJPH.2013.05.04.Art040

ISSN: $2520-3134$

[15]. Iwelunmor, J. E. (n.d.). Socio-Cultural Factors Influencing the Prevention of Mother-to-Child Transmission of HIV in Nigeria: A Synthesis of The Literature. Lagos: Not Published.

[16]. Kaida, A. Et. al. (2011). Childbearing Intentions of HIV-Positive Women of Reproductive Age in Soweto, South Africa: The Influence of Expanding Access to HAART in an HIV Hyper Endemic Setting. Not Published.

[17]. Kannaippan, S. Et. al. (2012). Desire for Motherhood: Exploring HIV-Positive Women's Desire, Intentions and Decision-Making in Attaining Motherhood. AIDS CARE.

[18]. Kasenga, F. (2010). Making it Happen: Prevention of Mother to Child Transmission of HIV in Rural Malawi. Retrieved from Global Health Action: http://www.globalhealthaction.net/index.php/gha/article/view/370

[19]. Kawale, P. Et. al. (2015). Providers Attitudes about Childbearing and Knowledge of Safer Conception at Two HIV Clinics in Malawi. Not Published.

[20]. King, Et. al. (1995). A Family Planning Intervention to Reduce Vertical Transmission of HIV in Rwanda. BMC Public Health, 45-51.

[21]. Leedy, P. D. (2010). Practical Research: Planning and Design. (9th ED). Boston: Pearson.

[22]. Maier, M. Et. al. (2008). Antiretroviral Therapy is Associated with Increased Fertility Desire, but not Pregnancy or Live Birth, Among HIV+ Women in an Early HIV Treatment Program in Rural Uuganda. Kampala: Epub. Ministry of Health (2011). Clinical Management of HIV in Children and Adults: Malawi Intergrated Guidelines for Providing HIV Services.

[23]. Ministry of Health (2014). Clinical Management of HIV in Children and Adults. Lilongwe: Ministry of Health. [24]. Newman, W. L. (1994). Social Research Methods. London: Boston Allyn and Bacon.

[25]. Njunga J. Et. al. (2010). The Divorce Program: Gendered Experiences on HIV Positive Mothers Enrolled in PMTCT Programs: The Case of Rural Malawi.

[26]. Nyasulu, J. Et. al. (2011). Decision Making for Women to Access Prevention of Mother to Child Transmission Services in Blantyre and Balaka Malawi. Journal of rural and Tropical Public Health. vol. 10, 95-100.

[27]. Patton, M. Q. (1990). Qualitative E valuation and Research Methods. (2nd Ed). California: Sage Publications. [28]. Polit, D. F. (2010). Nursing Research: Appraising Evidence for Nursing Practice. (8th Ed). Philadelphia: Lippicott Williams \& Wilkins.

[29]. Rutenberg, N. Et. al. (2000). Reproductive Decision-Making in the Context of HIV and AIDS: A Qualitative Study in Ndola, Zambia. Ndola: International Family Planning.

[30]. Strauss, A. \&. (1998). Basic of Qualitative Research: Techniques and Procedures for Developing Grounded Theory (2nd Ed). London: Thousand Oaks.

[31]. WHO. (2011). HIV and Infant Feeding: Guidelines for Decision Makers. Geneva: WHO.

[32]. Wolcott, H. F. (1980). Ethnographic Research in Education. Washington DC: American Educational Research Association. 